\title{
Peer-Interaktionen und Peer-Beziehungen
}

\author{
Susanne Viernickel ${ }^{1}$ und Dörte Weltzien² \\ 1 Universität Leipzig \\ ${ }^{2}$ Evangelische Hochschule Freiburg
}

Kinder treffen in Institutionen der frühkindlichen Bildung, Betreuung und Erziehung regelmäßig auf andere Kinder. Mit Eintritt in die Krippe, Kita oder Kindertagespflege sind sie tagtäglich in Peer-Interaktionen involviert. Folgt man der Sichtweise, dass unser Wissen über Dinge, Menschen, Beziehungen und Praktiken, Regeln und Normen des Zusammenlebens sozial konstruiert ist, dann ist auch die Sozialwelt der Peers als Ort anzuerkennen, an dem sich diese Entwicklungen vollziehen. Die aufgeschichteten Erfahrungen, die Kinder dort machen, formieren sich $\mathrm{zu}$ sozialen Beziehungen und werden gleichzeitig für die Selbstrepräsentationen der Kinder wirksam.

Die bahnbrechenden Arbeiten von Michael Tomasello und seiner Arbeitsgruppe (2019) legen nahe, dass beim Menschen von einer biologisch bedingten Kooperationsfähigkeit und Sozialität ausgegangen werden kann. Ausgestattet mit einer speziellen Art der kulturellen Intelligenz, die sozial-kognitive Fähigkeiten und Motivationen zu Zusammenarbeit, Kommunikation und sozialem Lernen umfasst, können Kinder im Laufe ihrer Entwicklung zunehmend gemeinsame Absichten verfolgen und sich auf Standards und Regeln der Zusammenarbeit verpflichten. Sie zeigen bereits im ersten Lebensjahr Interesse an anderen Kindern, das zu aneinander ausgerichteten Verhaltensweisen führt und zu prosozialem Handeln, sozialem Spiel und Kooperation ausgebaut wird (vgl. Viernickel, 2013).

Diese evolutionär verankerte Disposition wird im Laufe der ontogenetischen Entwicklung durch kulturelle und soziale Erfahrungen überformt. Peers stellen dabei andere Erfahrungsräume bereit als Erwachsene, was in der Qualität der Beziehungen begründet liegt. Denn die Beziehungen zwischen Erwachsenem und Kind werden - anders als die Beziehungen zwischen Kindern - maßgeblich durch deren soziale Positionierungen in der generationalen Ordnung beeinflusst. Dadurch, dass Peers auf einem ähnlichen Entwicklungsstand stehen, dieselben Entwicklungsthemen bearbeiten, einen vergleichbaren sozialen Status besitzen und einander im Wesentlichen ebenbürtig sind (vgl. Siebholz \& Winter, 2020, S.421), zeigen sie im Kontakt miteinander spezifische Verhaltensweisen und erwerben bzw. festigen spezifische Kompetenzen. In relativ stabilen Gruppenkontexten bilden sich Freundschaftsbeziehungen, Gruppenstrukturen und -positionen sowie eine eigenständige Kinderkultur mit ihr eigenen Verfahren, Aushandlungsprozessen und Regeln aus (vgl. Corsaro, 2009).

Trotz der individuellen und sozialen Bedeutsamkeit von Kind-Kind-Interaktionen, Peer-Beziehungen und PeerGruppen werden diese im Vergleich zu Arbeiten, die sich der Interaktions- und Beziehungsqualität zwischen pädagogischen Fachkräften und Kindern widmen, in der frühkindlichen Bildungsforschung noch relativ selten untersucht und auf ihre Voraussetzungen und Wirkungen hin befragt. Forschungsbedarf besteht u.a. hinsichtlich der Beschreibung kinderkultureller Phänomene, Praktiken und Prozesse, der Analyse von Zusammenhängen zwischen Erfahrungen in der Peer-Sozialwelt und individueller Kompetenzentwicklung und der Identifikation von Gelingens- und Hemmfaktoren für den Aufbau entwicklungsdienlicher Peer- und Freundschaftsbeziehungen.

Die für dieses Schwerpunktheft ausgewählten Beiträge tragen dazu bei, dieses Forschungsdesiderat zu füllen. Einen rahmenden Überblick über den aktuellen Wissensstand zum Thema Peer-Beziehungen und Kindergruppen gibt Petra Schneider-Andrich. Sie berücksichtigt dabei sowohl Arbeiten, die einer individualen, vorrangig entwicklungspsychologisch orientierten Forschungsperspektive folgen als auch soziologische, sozialpsychologische und gruppenanalytische Theorien und Befunde zur Kindergruppe als Gesamtfigur.

Die drei folgenden empirischen Arbeiten eint neben der übergreifenden Thematik der Peer-Interaktionen und Peer-Beziehungen auch der methodische Zugriff über videografisch fixiertes Material. Dieses scheint somit - und zwar unabhängig von qualitativen oder quantitativen para- 
digmatischen Verortungen - für die Feinanalyse sprachlicher und nichtsprachlicher Handlungen und die Rekonstruktion komplexer sozialer Situationen besonders geeignet, wenngleich auch hier spezifische Limitationen zu diskutieren sind (vgl. Göbel, 2018, S.56ff.).

Der Beitrag von Stephanie Simon, Barbara Lochner und Werner Thole nimmt in den Blick, wie Kinder im Kontext institutioneller Arrangements Peer- und Freundschaftsbeziehungen zum Thema machen. Hierzu wurden die verbalen Ausführungen von Kindern im Kontext von mehr als 100 Videospaziergängen in 14 Kindertageseinrichtungen analysiert. Es zeigt sich, dass Peerinteraktionen und -beziehungen in institutionellen Arrangements für Kinder bedeutsam, präsent und kommunikativ explizierbar sind. Die Rekonstruktion der kindlichen Perspektiven verweist darüber hinaus auf die Vielgestaltigkeit und Komplexität von Beziehungserfahrungen in Kinderfreundschaften.

Kinder haben unterschiedliche Voraussetzungen, um sich erfolgreich in Peer-Interaktionen und Gruppenprozesse einzubringen, wobei im Kindergartenalter die sprachliche Verständigungsfähigkeit eine wichtige Rolle spielt. Aleksandra Syczewska und Ulla Licandro analysieren, wie Kinder mit unterschiedlichen Kompetenzprofilen im Hinblick auf ihren Zweitspracherwerb Peer-Interaktionen initiieren und welche Reaktionen von Seiten der Peers darauf erfolgen. Die Ergebnisse bestätigen frühere Befunde, dass Kinder mit geringen Kompetenzen in der Umgebungssprache einem höheren Risiko ausgesetzt sind, von ihren Peers ignoriert oder abgelehnt zu werden (von Grünigen, Perren, Nägele \& Alsaker, 2010). Die Autorinnen zeigen, dass Kinder vielfältige Initiierungsstrategien erfolgreich einsetzen, diese jedoch in Abhängigkeit von den Wortschatzkompetenzen in der Zweitsprache Deutsch stehen. Kinder mit geringeren Kompetenzen nutzen stärker nonverbale Strategien und erfahren häufiger ablehnende Reaktionen von Seiten der Peers.

Der vierte Beitrag des Schwerpunkthefts schließt an diese Befunde an und behandelt die Frage nach der Wirksamkeit gezielter pädagogischer Interventionen zur Unterstützung von Peer-Beziehungen in sprachlich heterogenen institutionellen Settings. Dabei nutzen Sonja Perren,
Isabelle Kalkusch, Ann-Kathrin Jaggy, Carine Burkhardt Bossi, Barbara Weiss und Fabio Sticca das soziale Fantasiespiel als Entwicklungskontext und untersuchen, inwiefern die Förderung der Fantasiespielqualität bei Kindern im dritten und vierten Lebensjahr zu einer Verbesserung ihrer Peer-Beziehungen beiträgt. In einem anspruchsvollen randomisierten kontrollierten Interventionsdesign mit drei Messzeitpunkten zeigte sich, dass die Fantasiespielqualität unabhängig vom kindlichen Sprachverständnis durch aktive Spielbegleitung gefördert werden kann. Je höher Fantasiespielqualität und Sprachverständnis waren, desto besser wurden die Kinder in ihrer Peer-Gruppe akzeptiert und desto seltener erfuhren sie viktimisierendes Verhalten von Seiten der Peers. Von einer Steigerung der Fantasiespielqualität profitierten dabei vor allem Kinder mit eingeschränktem Sprachverständnis.

\section{Literatur}

Corsaro, W. A. (2009). Peer Culture. In J. Qvortrup, W. A. Corsaro \& M.-S. Honig (Eds.), The Palgrave Handbook of Childhood Studies (pp.301 - 315). London: Palgrave Macmillan.

Göbel, S. (2018). Alltagspraktiken in Kindertageseinrichtungen. Eine videographische Studie zum Miteinander von pädagogischen Fachkräften und Kindern. Wiesbaden: Springer VS.

Siebholz, S. \& Winter, D. (2020). Peers in der frühen Kindheit. In R. Braches-Chyrek, C. Röhner, H. Sünker \& M. Hopf (Hrsg.), Handbuch Frühe Kindheit (2. aktual. Aufl.) (S.421-431). Opladen: Barbara Budrich.

Tomasello, M. (2019). Becoming Human. A Theory of Ontogeny. Cambridge, MA: Harvard University Press.

Viernickel, S. (2013). Soziale Entwicklung. In M. Stamm \& D. Edelmann (Hrsg.), Handbuch Frühkindliche Bildungsforschung (S.633-652). Wiesbaden: Springer VS.

von Grünigen, R., Perren, S., Nägele, C. \& Alsaker, F. D. (2010). Immigrant children's peer acceptance and victimization in kindergarten. The role of local language competence. British Journal of Developmental Psychology, 28, 679-697.

Susanne Viernickel Dörte Weltzien

susanne.viernickel@uni-leipzig.de weltzien@eh-freiburg.de 